\title{
Detection of Exudates Caused By Diabetic Retinopathy in Fundus Retinal Image Using Fuzzy K Means and Neural Network
}

\author{
T.Vandarkuzhali, ${ }^{1}$, C.S.Ravichandran ${ }^{2}$, D.Preethi ${ }^{3}$, \\ Assistant professor ${ }^{1}$,Dean of EEE ${ }^{2}, \quad P G$ Scholar ${ }^{3}$ \\ Hindusthan college of Engineering and Technology ${ }^{(1)}$, \\ Sri Ramakrishna Engineering college ${ }^{(2)}$, \\ Hindusthan college of Engineering and Technology ${ }^{(3)}$
}

\begin{abstract}
Image processing is unavoidable in modern ophthalmology, as it heavily dependent on visually oriented signs. The various diseases which will affect the eye can be found with the help of digital fundus image. In manual analysis, due to unavailability of the trained ophthalmologist, the diagnosis of retinal diseases becomes unclear. Thus, automated analysis of fundus image is very much essential and will help to facilitate clinical diagnosis. An automated system for the detection of various abnormalities due to diabetic retinopathy $(D R)$ in retinal image is presented here. Fuzzy logic and neural network is used to identify the abnormalities in the fovea. These are evaluated for both normal as well as affected retinal images. A high performance language for technical computing MATLAB, is used here to implement the concept.

Keywords- Diabetic Retinopathy, Fovea, Fundus retlnal image, Fuzzy K means, Feed Forward Neural Network.
\end{abstract}

\section{A.Human Eye}

\section{Introduction}

The human eye has been called the most complex organ in our body. Human eye is divided into three parts Anatomy : structure of an eye .Physiology: function of these structure Pathology: disease and disorder of these structure

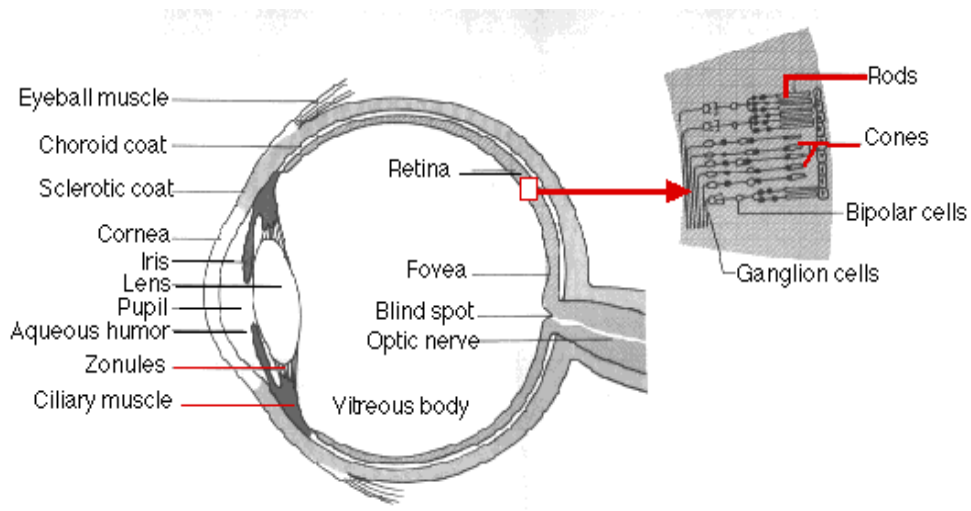

Fig. 1 Human Eye

The cornea is the clear front window of the eye that transmits and focuses light into eye. The iris is the coloured part of the eye that helps regulate the amount of light that enters the eye. The pupil is the dark aperture in the iris that determines how much light is let into the eye. The lens is the transparent structure inside the eye that focuses light rays onto the retina. The optic nerve is the nerve that connect the eye to the brain and carries the impulse formed by the retina to the visual cortex of the brain. The vitreous humour is a clear, jelly like substance that fills the middle of the eye .Macula is the centre most part of the retina and fovea is the centre of the macula

\section{$B$ Retina}

Retina converted light raise into electrical impulses and send toward brain through optic nerve Contain rods and cones. Rods are responsible for light and dark adaptation ,Cones are responsible for color vision[5] .Four kinds of light-sensitive receptors are found in the retina: rods and three kinds of cones, each "tuned" to respond best to light from a portion of the spectrum of visible light 


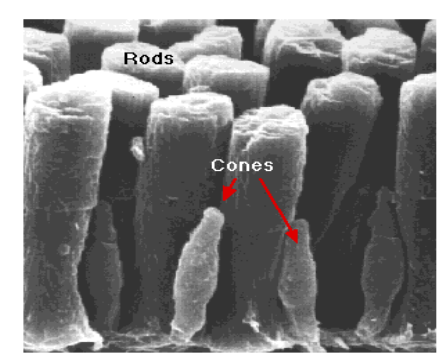

\section{Fundus Image}

Fig. 2 Rods and Cones

The colour fundus images are used to keep track of the eye diseases by ophthalmologist. Developing automatic fundus image analyzing and diagnosis. Extraction of the normal and abnormal features in colour fundus images is fundamental and useful to automatic understanding of fundus images. The normal features of fundus images include,opticdisk,fovea,blood,vessels,etc[2],[5],[6].and abnormal features include exudates, haemorrhages, microneurysms.

\section{$D$ Diabetes}

Diabetes is due to high sugar level in blood. There are two types of Insulin-dependent caused when beta cell in the pancreas get damaged develops it will be occur most frequently between 10 and 20 years of age. Non-insulin-dependent diabetes (NIDD): Type 2.It will be caused when cell of body resistance against insulin occur most frequently between the ages of 50 and 70 years. Diabetic retinopathy is a serious complication of diabetes.

\section{$E$ Diabetic Retinopathy}

Diabetic retinopathy, is retinopathy (damage to the retina) caused by complications of diabetes, which can eventually lead to blindness. It is an ocular manifestation of systemic disease which affects up to $80 \%$ of all patients who have had diabetes for 10 years or more. Despite these intimidating statistics, research indicates that at least $90 \%$ of these new cases could be reduced if there was proper and vigilant treatment and monitoring of the eyes. The longer a person has diabetes, the higher his or her chances of developing diabetic retinopathy.

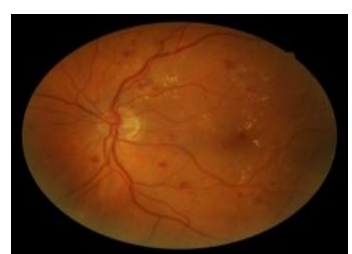

$\mathrm{T}$ here are three main type of retinopathy

Fig.3 Retina with Diabetic Retinopathy

Background retinopathy: Small red dots will appear on retina due to tiny swellings in the blood vessel walls. The number of microaneurysms, the little red dots the doctor sees, indicate the likelihood of more severe problems in the years to come. As the damage is mild at this stage, your sight will be nearly perfect. However, the condition does progress. If you have been diabetic 30 years, even with the best control, these may develop. But most people who have background retinopathy have not been diabetic that long, and need better control as per these targets.

BDR consists of:

- Microaneurisms: these are usually the earliest visible change in retinopathy seen on exam with an ophthalmoscope as scattered red spots in the retina where tiny, weakened blood vessels have ballooned out.

- Hemorrhages: bleeding occurs from damaged blood vessels into the retinal layers. This will not affect vision unless the bleeding occurs in or near the Macula.

- Hard Exudates: caused by proteins and lipids from the blood leaking into the retina through damaged blood vessels. They appear on the ophthalmoscope as hard white or yellow areas, sometimes in a ringlike structure around leaking capillaries. Again vision is not affected unless the macula is involved. 


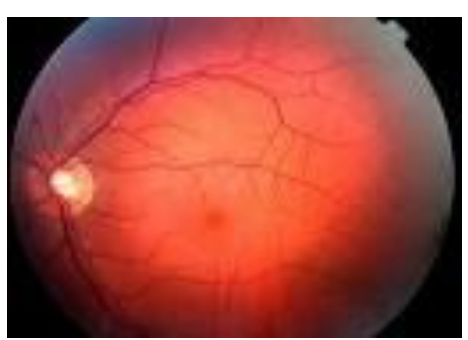

Fig.4 Background Retinopathy

Pre-proliferative retinopathy: Retina swells and leaks blood reading small print may become particularly difficult. In this condition the retina has been damaged by the higher than normal sugar levels over several years. The condition is called 'pre-proliferative' as it usually progresses to develop proliferative retinopathy, when 'new vessels' develop. It is now generally termed 'non-proliferative'.

In severe forms of pre-proliferative retinopathy there are lot of haemorrhages, as the retina is very ischaemic. This needs laser treatment to prevent new vessel growth. Proliferative retinopathy in one eye is especially likely if the other eye has already developed new vessels.

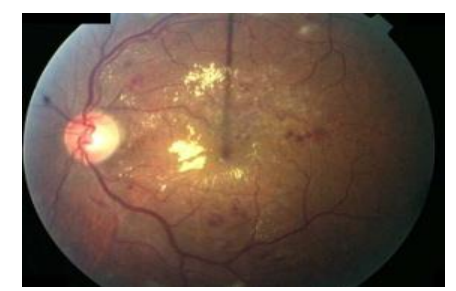

Fig.5 Pre-Proliferative retinopathy

Proliferative retinopathy: It is third stage of retinopathy usually causing a sudden loss of vision In this condition very small blood vessels grow from the surface of the retina.

The retina is the film at the back of your eye, and the tiny blood vessels are capillaries. These growing blood vessels are very delicate and bleed easily. Without laser treatment, the bleeding causes scar tissue that starts to shrink and pull the retina off, and the eye becomes blind. Lasertreatment prevents blindness, but often some vision is lost.

If you have had diabetes for years your retinae may develop this condition. As the retina is damaged by diabetes, the diseased retina releases special growth chemicals. These chemicals make tiny blood vessels grow: these are called 'new blood vessels'.

Another serious complication of proliferative diabetic retinopathy is neovascular glaucoma. This occurs when abnormal blood vessels grow on the iris and over the drainage system of the eye (the trabecular meshwork). This can lead to extremely elevated eye pressure, pain, redness, and severe vision loss.

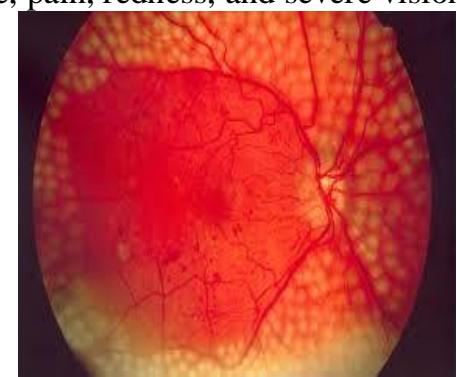

Fig.6.Proliferative Retinopathy

\section{F Exudates}

Exudates are accumulations of lipid and protein that oozes out of blood vessels due to inflammation and are deposited in nearby tissues[3]. They are typically bright, reflective, white or cream colored lesions seen on the retina . if this occurs on the macula, the vision may be lost

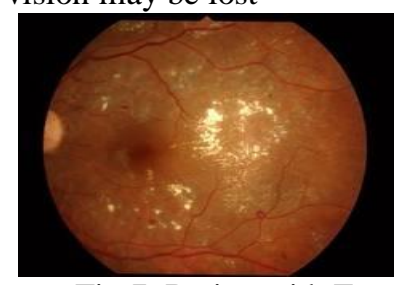

Fig.7. Retina with Exudates 


\section{Problem Formulation}

For non-invasive treatment, great effort from opthamologist is required. Diagnostics of retinal diseases include complex examination by retinologist and fundoscopy. In the existing method Fuzzy C Means is used which is not efficient in clustering. so, the Fuzzy K Means is used which is efficient in clustering .If the clustering is done efficiently, the diagnosis of the exudates will be accurate and the treatment can be done accordingly.

\section{Implementation}

In this paper, the exudates due to diabetic retinopathy is identified using the fuzzy $\mathrm{k}$ means

\section{$A$ Pre-processing done by Histogram Equalization}

Histogram equalization is a method in image processing of contrast adjustment using the image's histogram. This method usually increases the global contrast of many images, especially when the usable data of the image is represented by close contrast values. Through this adjustment, the intensities can be better distributed on the histogram. This allows for areas of lower local contrast to gain a higher contrast. Histogram equalization accomplishes this by effectively spreading out the most frequent intensity values. The method is useful in images with backgrounds and foregrounds that are both bright or both dark. In particular, the method can lead to better views of bone structure in x-ray images, and to better detail in photographs that are over or underexposed. Histogram equalization often produces unrealistic effects in photographs; however it is very useful for scientific images like thermal, satellite or x-ray images, often the same class of images that user would apply false-color to. The probability of an occurrence of a pixel of level $i$ in the image is

$$
p_{x}(i)=p(x=i)=\frac{n_{i}}{n}, \quad 0 \leq i<L
$$

Histrogram equalization of color image

This describes histogram equalization on a grayscale image. However it can also be used on color images by applying the same method separately to the Red, Green and Blue components of the RGB color values of the image. However, applying the same method on the Red, Green, and Blue components of an RGB image may yield dramatic changes in the image's color balance since the relative distributions of the color channels change as a result of applying the algorithm.

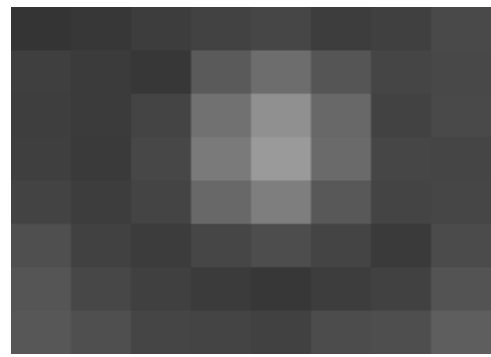

Fig.8 Original image

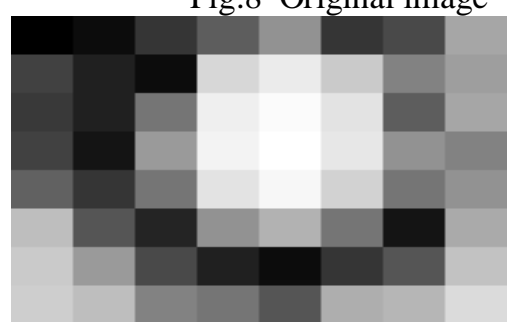

Fig.9 Equalized image

\section{B Fuzzy K Means}

Fuzzy K-Means (also called Fuzzy C-Means) is an extension of K-Means, the popular simple clustering technique. While K-Means discovers hard clusters (a point belong to only one cluster), Fuzzy KMeans is a more statistically formalized method and discovers soft clusters where a particular point can belong to more than one cluster with certain probability.

The algorithm is similar to k-means. Initialize $\mathrm{k}$ clusters until converged Compute the probability of a point belong to a cluster for every <point, cluster> pair Recomputed the cluster centres using above probability membership values of points to clusters. In data mining, $k$-means clustering is a method of cluster 
analysis which aims to partition $n$ observations into $k$ clusters in which each observation belongs to the cluster with the nearest mean. This results in a partitioning of the data space into Voronoi cells.

The problem is computationally difficult (NP-hard), however there are efficient heuristic algorithms that are commonly employed and converge quickly to a local optimum. These are usually similar to the expectationmaximization algorithm for mixtures of Gaussian distributions via an iterative refinement approach employed by both algorithms. Additionally, they both use cluster centers to model the data, however $k$-means clustering tends to find clusters of comparable spatial extent, while the expectation-maximization mechanism allows clusters to have different shapes.

The FKM algorithm is based on minimizing the following distortion:

$$
J_{q}(U, V)=\Sigma_{i} \Sigma_{k}\left(u_{i k}\right)^{q} d^{2}\left(X_{j}-V_{i}\right) ; K \leq N
$$

Compute the degree of membership of all feature vectors in all clusters:

$$
u_{i j}=\left[1 / d^{2}\left(X_{j}-V_{i}\right)\right]^{1 /(\mathrm{q}-1)} / \Sigma_{k}\left[1 / d^{2}\left(X_{j}-V_{i}\right)\right]^{1 /(\mathrm{q}-1)} \cdots \cdots
$$

Under the constraint: $\Sigma_{i} u_{i k}=1$ Compute new cluster prototypes $V_{i}$

$$
V_{i}=\Sigma_{j}\left[\left(u_{i j}\right)^{q} X_{j}\right] / \Sigma_{j}\left(u_{i j}\right)^{q}
$$

\section{Back Propagation Neural Network}

Back propagation is a common method of training artificial neural networks so as to minimize the objective function. and described it as a multi-stage dynamic system optimization method in . when applied in the context of neural networks and through the work of that it gained recognition, and it led to a "renaissance" in the field of artificial neural network research. It is a supervised learning method, and is a generalization of the delta rule. It requires a dataset of the desired output for many inputs, making up the training set The back propagation learning algorithm can be divided into two phases: propagation and weight update.

Phase 1: Propagation

Each propagation involves the following steps:

Forward propagation of a training pattern's input through the neural network in order to generate the propagation's output activations. Backward propagation of the propagation's output activations through the neural network using the training pattern's target in order to generate the deltas of all output and hidden neurons.

Phase 2: Weight update

For each weight-synapse follow the following steps:

- Multiply its output delta and input activation to get the gradient of the weight.

- Bring the weight in the opposite direction of the gradient by subtracting a ratio of it from the weight.

- This ratio influences the speed and quality of learning; it is called the learning rate.

The sign of the gradient of a weight indicates where the error is increasing; this is why the weight must be updated in the opposite direction.

Repeat phase 1 and 2 until the performance of the network is satisfactory.

\section{Results}

By using this software, automatic detection of exudates due to Diabetic retinopathy is achieved within short period of time. The accuracy and efficiency is increased when compared to Fuzzy C Means. Normal retinal images and affected images are used for the experiment. The following images were obtained when stimulated using MATLAB for normal and affected images.

\section{Input Image}

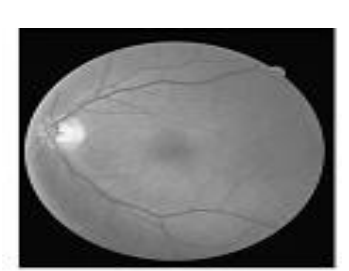

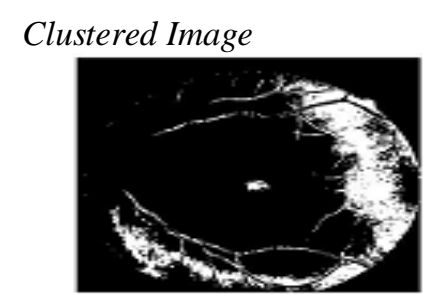

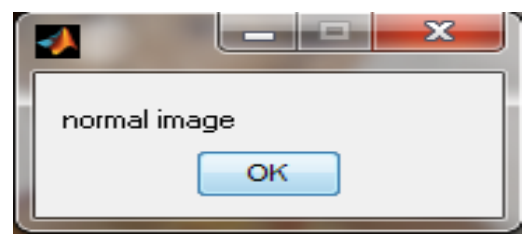

(a) 
Input Image
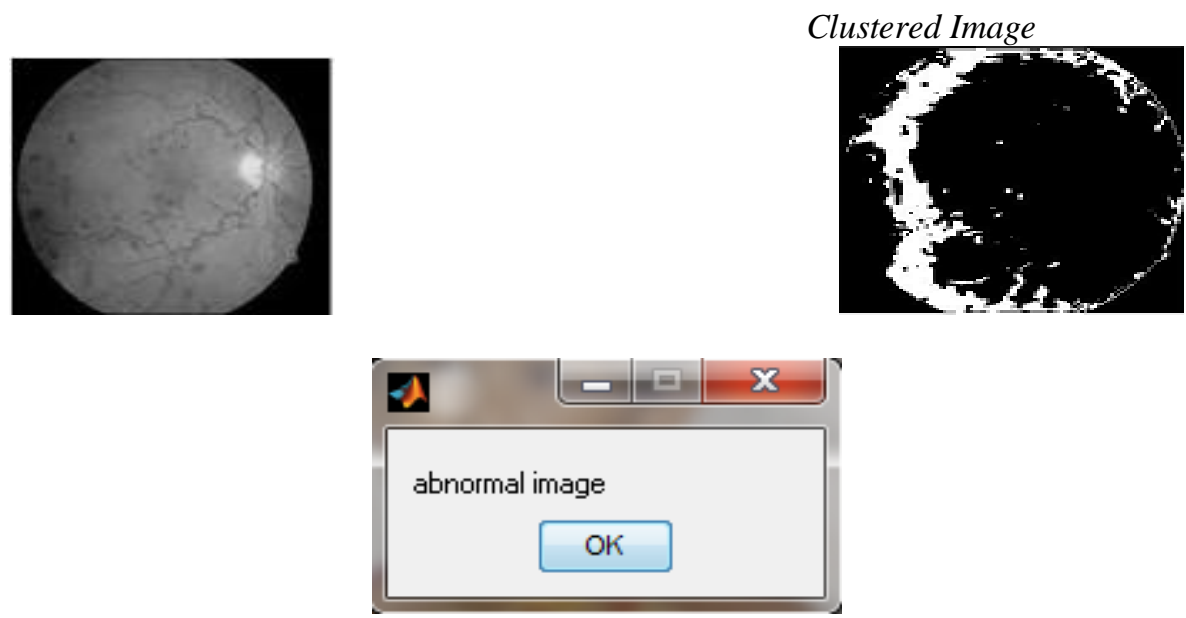

(b)

Fig.10 Input and Output images a)Norma Image B)Abnormal image

\section{Conclusion And Future Scope}

In the paper, an efficient method to identify the exudates due to diabetic retinopathy in fundus retinal images is described.FuzzyK means and the NeuralNetwork were used.This scheme is simple and efficient in extracting wheather the image is in normal or in abnormal stage. The extracted abnormal images may help in future diagnosis of related diseases.This work is performed for both normal and abnormal image. The extraction time for detecting diseases using Fuzzy K Means is very less when compared ti that of other technique.The continuation of this work is to be implemented in morphological method used to identify the hemorrages.

\section{Acknowledgement}

The authors would like to thank Dr. D.Poovannnan Assisant Doctor,Kovai Diabetes Specality Centre and Hospital for their valuable suggestions and help in obtaining the images used in the research.

\section{References}

[1] Adam Hoover* and Michael Goldbaum"Locating the Optic Nerve in a Retinal Image using the Fuzzy Convergence of the Blood Vessels"IEEE transcations on medical imaging, vol.22,no.8.august 2003

[2] Anderson Rocha *, Member, IEEE, Tiago Carvalho, Herbert F. Jelinek , Member, IEEE, Siome Goldenstein , Senior Member, IEEE, and Jacques Wainer"Points of Interest and Visual Dictionaries for Automatic Retinal Lesion Detection" 2244 IEEE transactions on biomedical engineering, vol. 59, no. 8, august 2012.

[3] Adam Hoover*, Valentina Kouznetsova, and Michael Goldbaum“Locating Blood Vessels in Retinal Images by Piecewise Threshold Probing of a Matched Filter Response" IEEE Transactions on medical imaging, vol. 19, no. 3, march 2000203

[4] F. Zana* and J. C. Klein“ A Multimodal Registration Algorithm of Eye Fundus Images.Using Vessels Detection and Hough Transform".IEEE Transactions on medical imaging, vol. 18, no. 5, may 1999419.

[5] Jeetinder Singh, Joshi G.D. Sivaswamy "Appearance based Object Detection in Colour Retinal Images" Proc. Of IEEE International conference on Images processing (ICIP), Oct 12-15,2008,San Diego,USA.

[6] Michal D. Abramoff, Senior Member ,IEEE, Monak k. Garvin, Member IEEE and Milan Sonak.Fellow, IEEE.“Retinal Imaging and Image Analysis" IEEE Reviews in Biomedical engineering, vol3,2010.

[7] Opas Chutatape, Senior Member, IEEE Proceedings of the $28^{\text {th }}$ IEEE EMBS annual International conference New york city, USA ,Aug 30-sept3,2006. ]"Fundus Foveal Localisation Based on Vessel Model"

[8] Soumitra Samanta,Sanjoy kumar sahaand bhabatose Chanda,1 ECSU, Indian.StatisticalInstitute,Kolkata,2 CSE department,jadavpur university, kolkata, india"A Simple and Fast Algorithm to Detect the Fovea Region in Fundus Retinal Images. 